\title{
Scaling of random spreading in small world networks
}

\author{
Jani Lahtinen ${ }^{1}$, János Kertész $^{1,2}$ and Kimmo Kaski ${ }^{1}$ \\ (October 31, 2018) \\ ${ }^{1}$ Laboratory of Computational Engineering, and Research Centre for Computational Science and Engineering, \\ Helsinki University of Technology, P.O.Box 9400, FIN-02015 HUT, Finland \\ ${ }^{2}$ Department of Theoretical Physics, Budapest University of Technology, Budafoki út 8, H-1111, Budapest, Hungary
}

\begin{abstract}
In this study we have carried out computer simulations of random walks on Watts-Strogatz-type small world networks and measured the mean number of visited sites and the return probabilities. These quantities were found to obey scaling behavior with intuitively reasoned exponents as long as the probability $p$ of having a long range bond was sufficiently low.

PACS numbers: 05.40.-a, 05.50.+q, 87.18.Sn
\end{abstract}

Recent interest in some disordered graphs was stimulated by a series of discoveries related to the so called small world properties of certain networks including social nets [1], document retrieval in the www [2], the internet [3], scientific cooperation relations [4] etc. (for reviews see [5]). Here 'small world' is used in the sense, that arbitrarily selected pairs of nodes can be reached from each other within few steps on the average, in spite of the relatively low number of links present in the system.

Many of the earlier network studies have dealt with the Watts-Strogatz small world network (WSSWN) model, which we will also study. We define it as a onedimensional ring consisting of $N$ nodes with $k$-neighbor interaction where, in addition, $N \cdot p$ new links are introduced between arbitrarily chosen, not yet connected nodes. Though the small world property is closely related to the dynamic process of information spreading in the network, relatively few papers have been devoted to this aspect [6] 9].

In the recent paper Jasch and Blumen [9] studied the target problem on a WSSWN model, i.e., the decay of the number of randomly distributed target sites on the graph where annihilating random walkers move around. There it turned out that this decay process is closely related to the mean number of distinct visited sites $S(n)$, where $n$ is the number of steps done by the walkers. In addition they observed the expected crossover in $S(n)$ from the $\sqrt{n}$ behavior that is characteristic for the one-dimensional case to $S(n) \propto n$ describing the high dimensional or random graph situation. $S(n)$ as a function of $p$ and $n$ has a scaling form:

$$
S(n)=n^{1 / 2} f\left(n p^{\alpha}\right)
$$

where $f$ is a universal scaling function with the following properties:

$$
f(x) \propto\left\{\begin{array}{rll}
\text { const } & \text { for } & x \ll 1 \\
\sqrt{x} & \text { for } & x \gg 1
\end{array}\right.
$$

Intuitively it is expected that $\alpha=2$ since in the system there exists a basic length scale $\xi \propto 1 / p$, characteristic of the average distance between nodes having long range links [7] for which the walker needs $n_{\xi} \propto \xi^{2}$ steps to sweep through. Thus the argument of the scaling function $f$ in Eq. (1) should be $n / n_{\xi}$. In spite of this strong argument Jasch and Blumen [9] found in their numerical simulations of WSSWN's a value $\alpha=1.85$. In the simulations they had chosen $N=50000$ by taking an average over 500 random walkers for each of the 100 WSSWN and they varied $p$ in the interval $0.01 \leq p \leq 0.1$. The main aim of the present note is to resolve the discrepancy in the value of $\alpha$.

It is first noted that Eq. (1), as is usual in scaling theory, is valid only asymptotically and in this case the scaling limit is $N \rightarrow \infty, n \rightarrow \infty$ and $p \rightarrow 0$. The scaling regime can be estimated from the variation of the mean vertex distance $\ell$ as a function of $p$ [7]: It turns out that $\ell k / N$ is a scaling function with the argument $x=p \cdot k \cdot N$ and of sigmoidal shape. This curve suggests that one can not expect good scaling for the above mentioned crossover, if $p \cdot k \cdot N \gg 100$. Therefore, it seems likely that in $[9]$ the investigated values of $p$ were not small enough to assure the proper scaling behavior $\left(p_{\min } \cdot k \cdot N=1000\right.$ in [9]).

For this reason we have carried out our simulations with considerably smaller values of $p$. In order to do so, we had to increase the system size as well. Hence we have studied WSSWN's with $k=2, N=10^{5}$ and for each value of $p=10^{-4}, 10^{-3.5}, 10^{-3}, 10^{-2.5}$ we did 100 realizations and had 100 random walkers per realization $\left(p_{\min } \cdot k \cdot N=20\right)$. In these simulations we measured the average number of distinct visited sites $S(n)$ as functions of $n$ and $p$, as depicted in Fig 1. In this plot it is seen that for the two largest values of $p$ saturation of $S(n)$ has set in. 


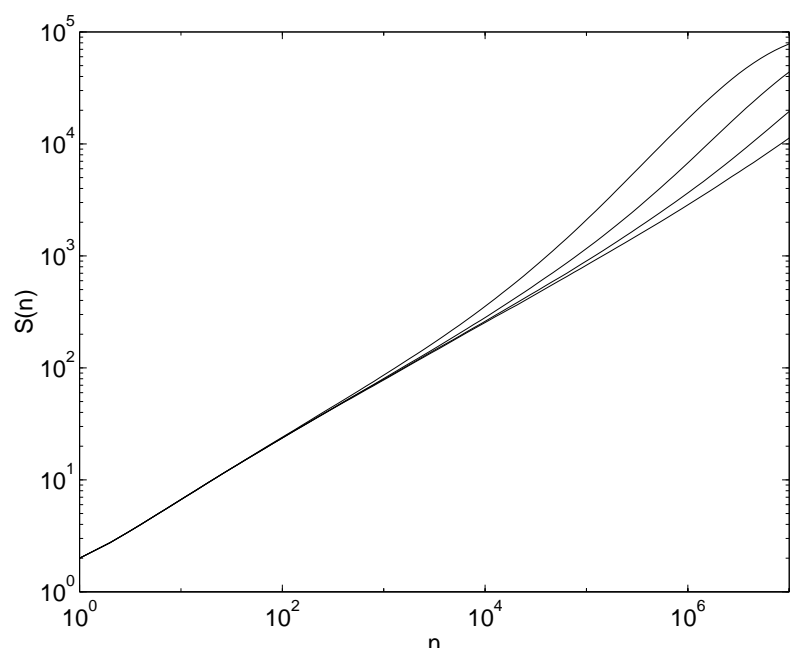

FIG. 1. Raw data for the average number of distinct sites visited $S$ as a function of time steps $n$ and the probability $p=10^{-4}, 10^{-3.5}, 10^{-3}, 10^{-2.5}$ increasing from bottom to top. For large $p$ the saturation due to the finite size $N=10^{5}$ of the systems is visible.

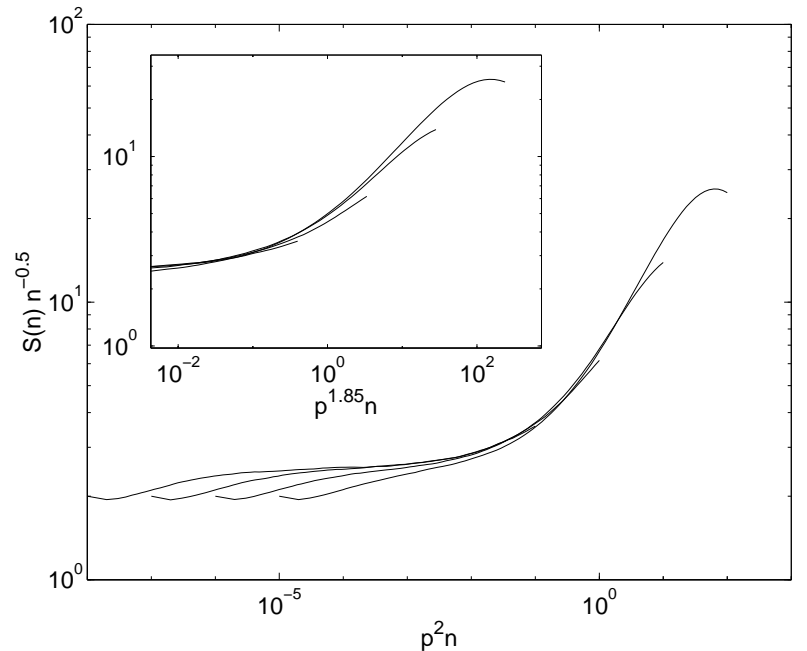

FIG. 2. Scaling plot of the data of Fig. 1 with $\alpha=2$. The inset shows the scaling plot with the exponent of [9].

Fig. 2 shows a scaling plot of the results on $S(n)$ where $S(n) / \sqrt{n}$ is plotted vs. $n p^{\alpha}$. The scaling was found to be optimal with the choise of $\alpha=2$. For comparison, we have also shown the same plot with $\alpha=1.85$, the value found in [9]. Our results clearly support the simple scaling picture discussed above.

We have also checked the scaling behavior for another quantity, namely the return probability $P_{00}(n)$. This is known to decay as $1 / \sqrt{n}$ for the $p=0$ case while an exponential decay is expected for large $p$. A scaling form similar to Eq. (1) should be valid for $P_{00}$ :

$$
P_{00}=n^{-1 / 2} \varphi(x)
$$

where $\varphi(x)$ is a rapidly decaying scaling function with the limit $\varphi=$ const. for $x \ll 1$. However, the argument of $\varphi$ should be the same as in eq. (1-2), namely $x=p^{\alpha} n$.

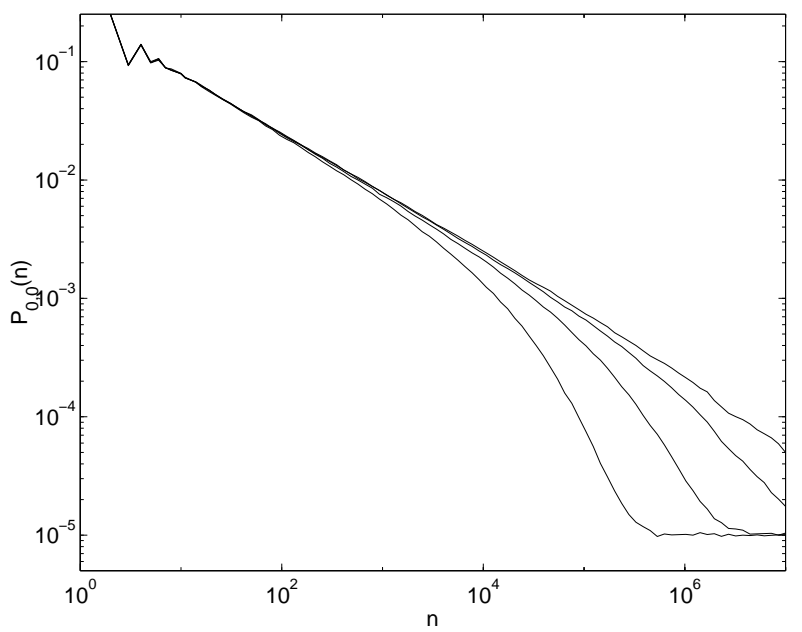

FIG. 3. Raw data for the return probability $P_{00}$. The $p$ values are the same as for Fig. 1, now increasing from top to bottom. The whole time interval was binned by 100 bins of equal sizes on the logarithmic scale.

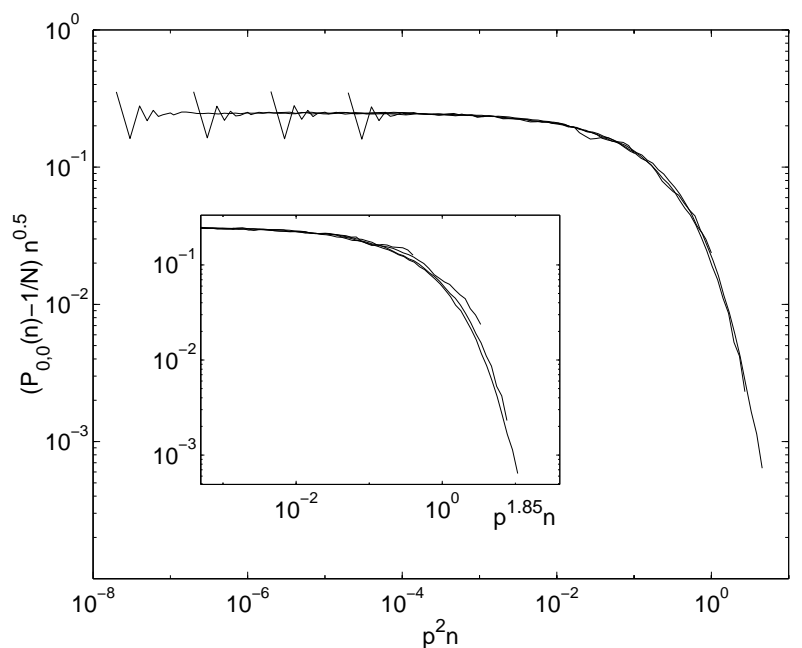

FIG. 4. Scaling plot of the data of Fig. 3 using $\alpha=2$. For minimizing the finite size effects the asymptotic value $1 / N=10^{-5}$ was subtracted from $P_{00}$. For comparison, the inset shows the scaling plot with the $\alpha$ of [9].

Here in the interest of even higher accuracy we have used 10 times more runs for the averages. In order to minimize the effect due to the finite size of the samples, we have subtracted $1 / N$, i.e., the $n \rightarrow \infty$ limit, from the measured values. Fig. 3 shows the data for the return probability $P_{00}$ and Fig. 4 the scaling plots. Again, we see that the scaling with the intuitively expected $\alpha=2$ is superior to the one obtained by the value of [9].

In conclusion we have shown that for sufficiently small probabilities of long range links the proper scaling vari- 
able for the average number of distinct sites visited and also for the return probability is $n p^{2}$, i.e., the natural exponent $\alpha=2$ holds for the Watts-Strogatz small world network.

This research was partially supported by OTKA T029985 and the Academy of Finland's Centre of Excellence programme, No. 44897 (Research Centre for Computational Science and Engineering). JK thanks for the kind hospitality of Laboratory of Computational Engineering at HUT.

[1] S. Milgram, Psychology Today 1, 61 (1967)

[2] R. Albert, H. Jeong, A. L. Barabási, Nature 401, 130 (1999)

[3] M. Faloutsos, P. Faloutsos and C. Faloutsos, Comput. Commun. Rev. 29, 251 (1999)

[4] S. Redner, Eur. Phys. J. B 4, 131 (1998), A.L. Barabási et al. cond-mat/0104162

[5] D. J. Watts: Small Worlds (Princeton, 1999), S.H. Strogatz: Nature 410, 268 (2001) R. Albert and A.L. Barabási: Rev. Mod. Phys. (in press)

[6] D. J. Watts, S. H. Strogatz, Nature 393, 440 (1998)

[7] M.E. Newman and D.J. Watts, Phys. Rev. E 60, 7332 (1999)

[8] C.F. Moukarzel, Phys. Rev. E 60, R6263 (1999), S.A. Pandit and R.E. Amitkar cond-mat/00004163

[9] F. Jasch and A. Blumen, Phys. Rev. E 63, 041108 (2001) 